\title{
Secondary Electron Spectral Changes of Irradiated Gold Nanoparticle Caused By PEGylation
}

\section{Morozov V.N. ${ }^{1}{ }^{1}$, , Belousov A.V. ${ }^{1}$, Krusanov G.A. ${ }^{3}$, Kolyvanova M.A. ${ }^{2}$, Chernyaev A.P. ${ }^{1,3}$, and Shtil A.A.4}

${ }^{1}$ Department of Physics, M.V. Lomonosov Moscow State University, Moscow, Russia

${ }^{2}$ A.I. Burnazyan Federal Medical Biophysical Center, Moscow, Russia

${ }^{3}$ D.V.Skobeltsyn Institute of Nuclear Physics at M.V. Lomonosov Moscow State University, Moscow, Russia

${ }^{4}$ N.N.Blokhin National Medical Center of Oncology, Moscow, Russia

\section{Abstract}

Gold nanoparticles attract attention for the use in radiation therapy of tumors due to the ability to enhance the efficacy of ionizing radiation. The magnitude of the radiosensitizing effect depends on the parameters of the nanoparticle, in particular

Corresponding Author:

Morozov V.N.

morozov.v.n@mail.ru

Received: 17 January 2018

Accepted: 25 March 2018

Published: 17 April 2018

Publishing services provided by Knowledge

(c) Morozov V.N. et al. This article is distributed under the terms of the Creative Commons

Attribution License, which

permits unrestricted use and redistribution provided that the original author and source are credited.

Selection and Peer-review under the responsibility of the PhysBioSymp17 Conference Committee.

\section{G OPEN ACCESS} on the modification of the surface. In the present work, the spectrum of secondary particles generated in a gold nanoparticle virtually irradiated with ${ }^{60} \mathrm{Co}$ gamma rays as a result of surface modification by a polyethylene glycol shell was studied. The Monte Carlo calculations revealed that modification of the nanoparticle's surface changes the spectrum of secondary particles. The most robust was the loss in low-energy electrons (51\%) whereas the yield of Compton electrons increased by 1.27 times. At the same time, no statistically significant changes were observed in the spectrum of secondary photons and photoelectrons. Simulation of the formation and distribution of secondary electron radiation makes it possible to evaluate the parameters important for the rational design of antitumor nanoradiosensitizers based on chemical elements with a high atomic number.

Keywords: gold nanoparticles, radiosensitizers, Monte-Carlo simulation, Geant4, radiation therapy, malignant tumors.

\section{Introduction}

Nanoparticles (NPs) of high-Z elements (e.g., gold (GNPs): $Z_{A u}=79$ ) are perspective candidates for the design of antitumor radiosensitizers because of their large cross section with photons [1]. GNPs are the most promising due to their physicochemical properties and high biocompatibility. GNPs absorb the energy of ionizing radiation and generate secondary particles such as photo-, Compton and ionizing electrons. These particles distribute their energy in different areas depending on LET. The low energy, 
short-range ionizing electrons (Auger, Coster-Kronig and fluorescent ones) deposit very high energies in the close proximity to the GNP surface. The size, shape and surface coating of NPs can influence the radiosensitization effect. For targeted delivery to the tumor and prevention of aggregation in high ionic strength media, the surface of NP can be modified by different agents including polyethylene glycol (PEG). Although PEGylated GNP have demonstrated the effect of radiosensitization, the impact of NP surface coating is poorly understood. This effect depends on coating thickness [2]; also, surface functionalization can dramatically decrease the production of radicals [3]. Furthermore, surface coating modifies dose distribution around GNP [4]. Despite these facts the Monte-Carlo studies frequently disregard GNP surface coatings. In the present work the effect of PEGylation on the formation of a secondary radiation spectrum emitted by a GNP under the action of gamma radiation was investigated by MonteCarlo simulation.
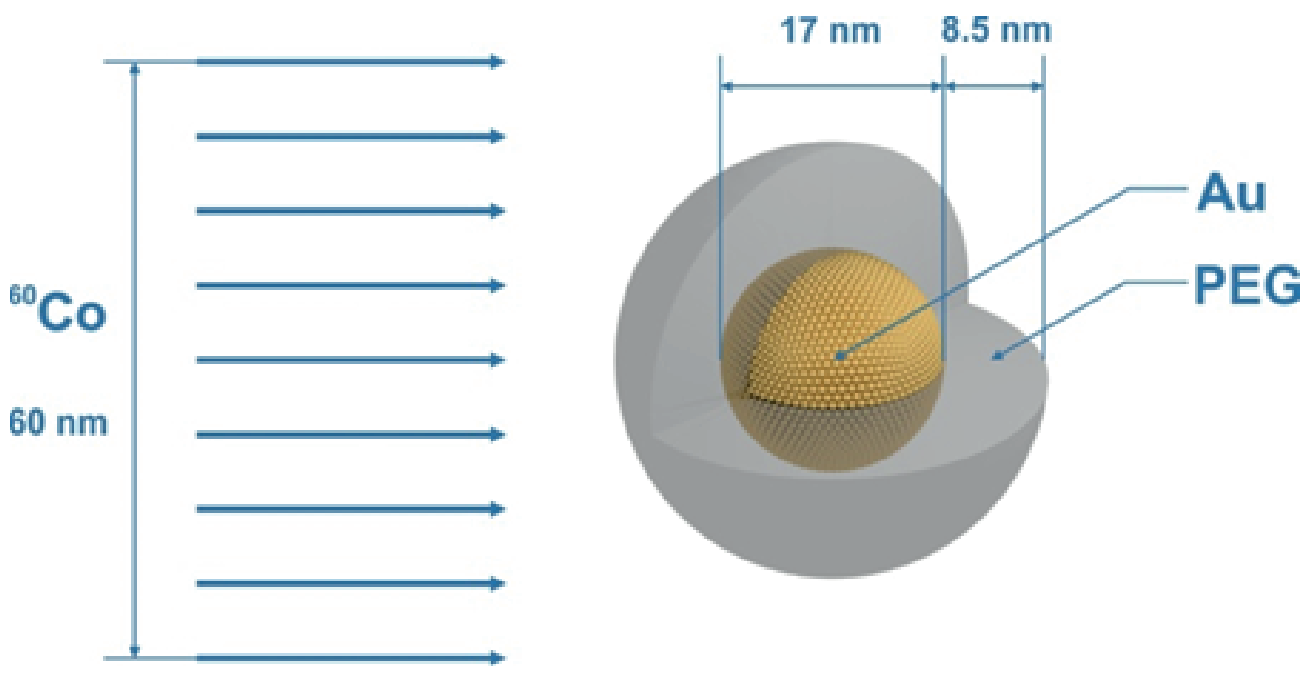

Figure 1: Scheme of Monte-Carlo simulations.

\section{Materials and methods}

We performed a Monte-Carlo simulation (a Geant4 code) to investigate the influence of ionizing radiation on low energy secondary electrons. A $17 \mathrm{~nm}$ GNP was modified with the PEG shell (thickness $8.5 \mathrm{~nm} ; M_{P E G}=5000 \mathrm{~g} / \mathrm{mol}$ ) [5]. GNP were virtually irradiated with a circle photon beam $(D=60 \mathrm{~nm})$ using a ${ }^{60} \mathrm{Co}$ source (1.17 MeV and 1.33 MeV) (Figure 1). The amounts of secondary electrons that left the gold core and the PEG shell were recorded and analyzed. 


\section{Results and discussions}

Due to the high atomic number $\left(Z_{A u}=79\right)$ the GNPs absorbs the energy of ionizing radiation more effectively then low-Z biological tissues. In turn, GNPs generate secondary radiation which consists of secondary particles: photoelectrons, Compton and ionization electrons (Auger, Coster-Kronig, fluorescence). Upon irradiation the two processes occur within the shell: 1) energy absorption of secondary radiation, generated by interaction of primary photons with NP gold core; 2 ) generation of secondary radiation caused by interaction of primary photons with the PEG shell. These processes depend on shell's material and energy of primary photons. At high photon energies, the number of absorbed low-energy electrons significantly exceeds the number of electrons generated in the envelope.

Secondary photons have a much larger mean free path than low-energy secondary electrons, so the absorbed dose created around the NP is formed mainly by electrons. Thus, secondary electrons are responsible for the radiosensitizing effect of NP.
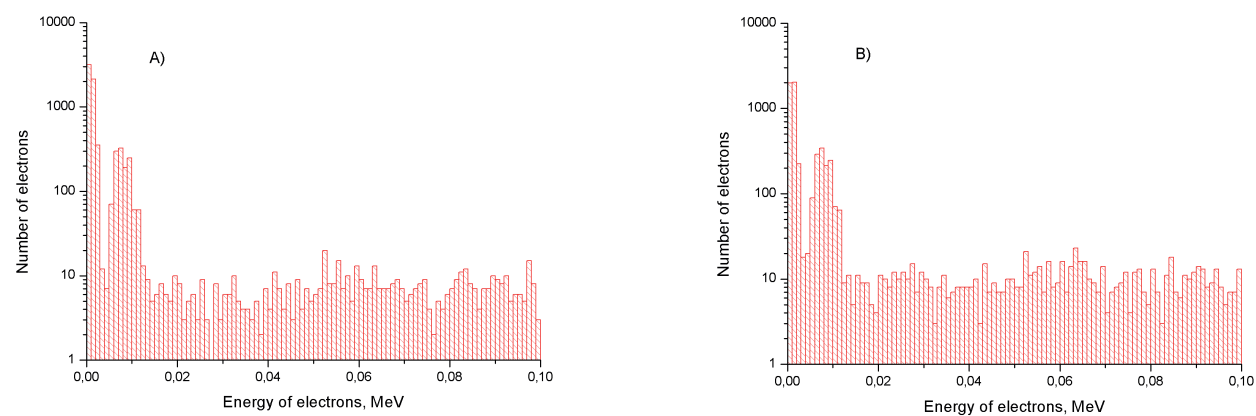

Figure 2: Secondary energy spectra of two models: A) without PEG shell; B) with PEG shell.

We found that the PEG shell is critical for spectral characteristics of low-energy ionization electrons and Compton electrons (Figure 2). No changes were revealed for photoelectrons and secondary photons. These phenomena are explained by the fact that ionization electrons have low energies and are more effectively retained inside the shell, especially when their mean free path is comparable to the shell's thickness. Even though PEG consists of light chemical elements, the shell forms an additional barrier for low energy electrons. To initiate water radiolysis, a key event in tumor cell death by radiation, electrons have to leave not only the gold core but also the PEG shell. About one half $(51 \%)$ of low-energy ionizing electrons generated in the gold core was absorbed by the PEG shell. As a result, ionization electrons lost $\sim 35 \%$ of their total energy. 
The number of photoelectrons varied insignificantly since their large kinetic energy and a range prevent the absorption in the shell. On the other hand, the Compton scattering prevails in the interaction of primary photons with the shell material. As a result, more Compton electrons (1.27-fold) were generated within the shell, thereby significantly increasing the overall electrons yield from the nanoparticle.

\section{Conclusions}

Surface coating is an integral part of the design of NPs for in vivo use. The spectrum of secondary electrons upon ${ }^{60} \mathrm{Co}$ irradiation of PEGylated (thickness $8.5 \mathrm{~nm} ; M_{P E G}=5000$ $\mathrm{g} / \mathrm{mol}$ ) GNP were investigated by Monte-Carlo simulation. More than half ( $51 \%$ ) lowenergy ionization electrons were absorbed by the PEG shell. The yield of Compton electrons increased 1.27-fold. No changes were revealed for photoelectrons and secondary photons. Thus, surface decoration, an important component of the functional design of nanoradiosensitizers, is meaninglful both for interaction with biological objects and for modification of dose distribution.

\section{Acknowledgment}

The authors are grateful to V.M. Rudoi, O.V. Dement'eva and L.A. Ilyin for critical discussions.

\section{References}

[1] J.F. Hainfeld, D.N. Slatkin, and H.M. Smilowitz, "The use of gold nanoparticles to enhance radiotherapy in mice," Physics in Medicine \& Biology, vol. 49, no. 18, Pp.309-315, 2004.

[2] M. Gilles, E. Brun, and C. Sicard-Roselli, "Gold nanoparticles functionalization notably decreases radiosensitization through hydroxyl radical production under ionizing radiation," Colloids and Surfaces B: Biointerfaces, vol. 123, pp. 770-777, 2014.

[3] C. Spaas, R. Dok, O. Deschaume, B. De Roo, M. Vervaele, J.W. Seo, C. Bartic, P. Hoet, F. Van den Heuvel, S. Nuyts, J.P. Locquet, "Dependence of Gold Nanoparticle Radiosensitization on Functionalizing Layer Thickness," Radiation Research, vol. 185, no. 4, pp. 384-392, 2016. 
[4] B. Koger, and C. Kirkby, "Dosimetric effects of polyethylene glycol surface coatings on gold nanoparticle radiosensitization," Physics in Medicine \& Biology, vol. 62, pp. 8455-8469, 2017.

[5] V.V. Terekhin, I.N. Senchikhin, O.V. Dement'eva, and V.M. Rudoy, "Conjugates of gold nanoparticles and poly(ethylene glycol): Formation in hydrosol, direct transfer to organic medium, and stability of organosols," Colloid Journal, vol. 7, no. 4, pp. 511519, 2015. 